\title{
Charge Localization in Disordered Colossal-Magnetoresistance Manganites
}

\author{
Qiming Li ${ }^{1}$ Jun Zang, ${ }^{2}$ A. R. Bishop ${ }^{2}$ and C. M. Soukoulis ${ }^{1}$ \\ ${ }^{1}$ Ames Laboratory-USDOE and Department of Physics and Astronomy, \\ Iowa State University, Ames, IA 50011 \\ ${ }^{2}$ Theoretical Division and Center for Nonlinear Studies \\ Los Alamos National Laboratory, MS B262, Los Alamos, NM 87545
}

(November 3, 1996)

\begin{abstract}
The metallic or insulating nature of the paramagnetic phase of the colossal-magnetoresistance manganites is investigated via a double exchange Hamiltonian with diagonal disorder. Mobility edge trajectory is determined with the transfer matrix method. Density of states calculations indicate that random hopping alone is not sufficient to induce Anderson localization at the Fermi level with 20-30\% doping. We argue that the metal-insulator transtion is likely due to the formation of localized polarons from nonuniform extended states as the effective band width is reduced by random hoppings and electron-electron interactions.
\end{abstract}

72.15.Gd, 72.15.Rn, $\quad 71.50 .+\mathrm{t}, \quad 71.55 .+\mathrm{Jv}, \quad 71.38 .+\mathrm{i}$

The recent discovery of colossal magnetoresistance (CMR) [1] in Mn-oxides $\mathrm{La}_{1-x} \mathrm{~A}_{x} \mathrm{MnO}_{3}$ (where $\mathrm{A}$ can be $\mathrm{Ca}, \mathrm{Sr}$, or $\mathrm{Ba}$ ) has generated extensive interests in these perovskites. The essential correlation between the magnetization and resistivity [2] in these compounds was well explained by the double exchange (DE) mechanism [3]. However, the precise nature of the ferromagnetic metal to paramagnetic insulator transition is still not well understood. Recently, Millis et al [4] argued that Jahn-Teller (JT) effects [5] have to be included to explain the observed resistivity behavior and the magnetic transition temperature. Millis et al [6] and Bishop and coworkers [7] then proposed that the metal-insulator transition (MIT) is a consequence of the large to small polaron transition, induced by the reduction of effective hopping integrals at temperatures near $T_{c}$. However, there are competing proposals 8,9 which advocate that the DE mechanism alone can explain the magnetic properties and MIT in the Mn-oxides. Notably, Varma [9] argued that random hopping in the paramagnetic phase due to the DE mechanism is sufficient to localize electrons and induce a MIT at $T_{c}$. Although there are mounting experimental evidences of lattice effects [10] and small polaron dynamics above $T_{c}$ 11, it is still not clear at the present whether the small polaron formation for temperatures close to $T_{c}$ is the driving force of the MIT or it is merely a consequence of the MIT from the DE mechanism itself. To investigate the origin of the MIT, a precise calculation of the localization effects in the DE model is needed. In this Communication we study the localization properties in the DE model using the well-developed transfer matrix method 12].

The DE mechanism can be described by the following Hamiltonian:

$$
H=\sum_{\langle i j\rangle}\left(t_{i j}^{\gamma \gamma^{\prime}} c_{i \gamma \sigma}^{\dagger} c_{j \gamma^{\prime} \sigma}+\text { h.c. }\right)+\sum_{i}\left(\epsilon_{i \gamma}-\mu\right) n_{i \gamma}
$$

$$
-J_{H} \sum_{i} \vec{\sigma}_{i \gamma} \cdot \vec{S}_{i}+V \sum_{i} n_{i a} n_{i b}
$$

where $\vec{\sigma}_{i \gamma}$ is the electronic spin operator and $\vec{S}_{i}$ is the local spin $S=3 / 2$ of three $\mathrm{Mn} t_{2 g} d$-electrons. The operators $c_{i \gamma \sigma}\left(c_{i \gamma \sigma}^{\dagger}\right)$ annihilate (create) a mobile $e_{g}$ electron with spin $\sigma$ at orbital- $\gamma(\gamma \in\{a, b\}) . n_{i \gamma}=c_{i \gamma \sigma}^{\dagger} c_{i \gamma \sigma}$. The random diagonal energy $\epsilon_{i \gamma}$ is included to account for substitutional disorders [13]. The $J_{H}$-term describes the Hund's rule coupling between the local spin of $t_{2 g}$ electrons and the $e_{g}$ electrons; the $V$-term describe the on-site inter-orbital electron-electron (e-e) interactions. In the Mn-oxides, $J_{H} \gg t / S$. Here we neglect the on-site intra-orbital e-e interactions and exchange interactions, which is reasonable in the large $J_{H}$ limit.

We consider only the limit $S J_{H} / t \rightarrow \infty$. As a consequence, the electronic spin at each site is parallel to the local spin, for the low energy states of interest. To study these low energy states, we can use a projection operator $\mathcal{P}$ to project out the high energy states. Then the Hund's rule coupling interaction can be dropped and the hopping term is renormalized to

$$
t_{i j}=t\left[\cos \left(\frac{\theta_{i}}{2}\right) \cos \left(\frac{\theta_{j}}{2}\right)+\sin \left(\frac{\theta_{i}}{2}\right) \sin \left(\frac{\theta_{j}}{2}\right) e^{i\left(\phi_{i}-\phi_{j}\right)}\right]
$$

in the classical spin limit $S=\infty$, where $\left(\theta_{i}, \phi_{i}\right)$ is the polar angles of the classical spin at site- $i$. Both finite $J_{H}$ and $S$ will decrease the localization effect, while the inter-orbital e-e interaction $V$ will probably increase it. To simplify the problem further, we will neglect the ee interaction here and assume that its only important contribution is to renormalize Hamiltonian parameters. The Hamiltonian (11) is then transformed to an effective one-electron Hamiltonian of the form

$$
H=\sum_{i}\left(\epsilon_{i}-\mu\right) n_{i}+\sum_{<i j>}\left(t_{i j} c_{i}^{\dagger} c_{j}+H . c .\right) .
$$


The Hamiltonian (3) describes the dynamics of electrons in a static background of classical spins, with the hopping matrix elements dependent on the spin configuration via Eq.(2). This approximation of dynamic disorder by static disorder is valid in the large $S$ limit. Note that we also replace the two-orbitals by one effective orbital. If there is no Jahn-Teller coupling, the carrier density of each orbital would be approximately half of the doping $x$. However, static or dynamic JT effects will complicate this issue and the exact correspondence is not well understood at the metallic region at present.

In the simplified Hamiltonian (3), electron localization is due to both off-diagonal and diagonal disorder. The off-diagonal disorder is intrinsic to the DE model in the paramagnetic phase, and the resistivity due to this spin-disordered scattering was calculated in Bornapproximation [14] or memory function approximation [4]. Localization via off-diagonal disorder has not been studied extensively. A few calculations 15 have been done for uniform distributions of real hopping integrals $t$ in one and two-dimensional systems. Also there are studies on $2 \mathrm{D}$ random flux systems with uniform $|t|[16]$. The most relevant work for the present model was a study by Economou and Antoniou [17] for systems with a semicircle distribution of hopping integrals. Their work was based on the localization criteria of Economou and Licciardello 18]. A recent calculation 19 based on the $\mathrm{Zi}$ man criterion 20] was also reported. None of these calculations produce the precise mobility edge. Moreover, the random Berry's phases (the phase of $t_{i j}$ in Eq.(2)) are not included, and their importance is difficult to assess.

Here we investigate the localization properties of Hamiltonian (3) with the transfer matrix method [12]. This technique, coupled with finite size scaling analysis, produces the most reliable information about the extended or localized nature of the eigenstates. In this technique, one considers a bar of length $N$ and cross section $M \times M$. One determines the largest localization length $\lambda_{M}$ as $N \rightarrow \infty$ from the smallest Lyapunov coefficient of the product of the random transfer matrix relevant to Eq.(3). The nature of the eigenstate can be determined by studying the scaling property of the localization length of finite systems. For extended (localized) states, $\lambda_{M} / M$ increases (decreases) with increasing $M$. At the mobility edge, which separates the extended from the localized states, $\lambda_{M} / M$ is independent of $\mathrm{M}$ and this behavior defines the Anderson transition.

In the paramagnetic phase, the direction of the local spin $\vec{S}_{i}$ is chosen to be uniformly distributed on a sphere: $P\left(\phi_{i}\right)=1 /(2 \pi) ; P\left(\cos \left(\theta_{i}\right)\right)=1 / 2$. Once the spin configuration $\left\{\vec{S}_{i}\right\}$ is specified, the nearest neighbor hopping integrals can be obtained via Eq.(2). The random diagonal site-energy $\epsilon_{i}$ is assumed to be distributed uniformly between $[-\mathrm{W} / 2, \mathrm{~W} / 2]$. In our calculation, we have used systems with widths $M=4-14$ and the length $\mathrm{N}$ on the order of $\mathrm{N}=30,000$ to minimize errors.

We first investigate the localization effects in the absence of any diagonal disorder, $\mathrm{W}=0$, to see whether random hoppings with Berry phases in the paramagnetic phase is alone sufficient to lead to localization of a large fraction of electrons, as has been argued in [9]. Our results are presented in Fig. 1, in which we plot the ratio of the calculated finite size localization length $\lambda_{M}$ with M, as a function of $\mathrm{M}$ for different energies. It is clear that the mobility edge $\mathrm{E}_{c}$, where $\lambda_{M} / M$ should be a constant independent of $\mathrm{M}$, is located between $3.55<\left|E_{c}\right| / t<3.6$. To determine whether the Fermi level is below or above $\mathrm{E}_{c}$ at certain doping levels, we need to know the density of states (DOS) of the system, which we obtain by directly diagonalizing the Hamiltonian matrix for a finite size $(10 \times 10 \times 10)$ cluster. The integrated DOS and the DOS, averaged over many (100) configurations, are shown in Fig. 2. This indicates that less than $0.5 \%$ of states are below $E_{c}=-3.56 t$ (Fig. 2), far less than the required $20-30 \%$ for the CMR system. Therefore, the present calculation confirms the suggestion [6] that purely off-diagonal disorder in the DE model is far from sufficient to localize electrons in the CMR materials at the 20-30\% doping level. The inefficiency of the offdiagonal disorder can be understood by looking at the distributions of the amplitude of the hopping integral $t_{i j}$, $P\left(\left|t_{i j}\right|\right)=2\left|t_{i j}\right| / t^{2}$. This distribution has small weight for small $\left|t_{i j}\right|$ which are most important for localization. The presence of the Berry phase, on the other hand, weakens further the localization effect by breaking the timereversal symmetry 21]. In fact the mobility edge without the Berry phase is located at $\left|E_{c}\right| \approx 3.3 t$. The inefficiency of the pure DE model to localize electrons clearly points towards the necessity to include other effects, such as JT electron-phonon coupling, electron-electron interaction effects, and diagonal substitutional disorders.

To investigate the effect of diagonal disorders, we have calculated $\lambda_{M}$ for different values of $\mathrm{W}$ and $\mathrm{E}$, similar to the $\mathrm{W}=0$ case, to determine the location of the mobility edge at fixed $\mathrm{W}$. The mobility edge trajectories in the W-E plane is shown as in Fig. 3. The shape of the mobility edge bears remarkable resemblance to the mobility edge trajectory in the Anderson model with diagonal disorder alone [22]. The main difference is in the energy scale which is smaller in the present system due to the smaller average value of the hopping integrals. For the DE model, we obtain $\left\langle\left|t_{i j}\right|\right\rangle=\frac{2}{3} t$, leading to an effective band edge [23] at $E_{b}=-4.0 t$ at $\mathrm{W}=0$. This reduction of band width also accounts for the smaller critical disorder $W_{c}$ at the band center. The outward shift of the mobility edge for small $\mathrm{W}$ is due to the increase of the effective band width with $\mathrm{W}$. If $\mathrm{E}$ is normalized with the effective band width instead of $t$, the region of extended states will always shrink with increasing disorder W. In the same figure, we have also plotted the equal-integrated-DOS lines. Figure 3 shows that to achieve localization of $20-30 \%$ of 
the electrons, the presence of a substantial amount of diagonal disorders $\mathrm{W}$, in the range of 10-12 in units of $t$, is required.

The ture mobility edge is controlled by the formation of localized polarons in systems with strong electronphonon couplings. The presence of disorder changes the character of the polaron formation in three dimensions. In ordered systems, an abrupt change of the polaron state from nearly free type (large polaron) to self-trapped type (small polaron) occurs as the electron-phonon coupling reaches a critical value [24]. In disordered systems, however, localized polaron of intermediate sizes can form from the nonuniform extended electronic states above the mobility edge even with moderate coupling [25]. This transition occurs at $\xi \approx 10 \lambda^{-2 / 3}$, where $\lambda$ is the dimensionless electron-phonon coupling constant. The true mobility edge, obtained by using the coherent length $\xi$ from the finite size scaling analysis to be discussed below, is indicated in Fig. 3, for $\lambda=0.03$ and 1. For large values of coupling constant, $\lambda>>1$, small polaron picture prevails. Figure 2 and 3 are the principal results of this work.

The presence of the Berry phase breaks time-reversal symmetry, and hence the present model belongs to the unitary universality class. The critical property around the MIT is investigated using the one-parameter-scaling procedure [12]. We have been successful (Fig. 4) in placing all our data on the same universal scaling curve, $\lambda_{m} / M=f(\xi / M)$, where $\xi(W, E)$ is the scaling parameter corresponding to the infinite size localization and correlation length in the localized and metallic regimes respectively. Moreover, our scaling data fall on the scaling function (shown as solid line in fig. 4) of the standard Anderson model with diagonal disorder alone. The critical exponent $\nu$ for the localization and correlation lengths, $\xi \sim\left|E-E_{c}\right|^{-\nu}$, is found to be $\nu=1.0 \pm 0.2$, consistent with the value for the standard Anderson model [12. Our result further supports previous conclusions 26, 27 that the critical property of the disorder-induced MIT is not modified by broken time-reversal symmetry in three dimensional systems.

In the Mn-oxides $\mathrm{La}_{1-x} \mathrm{~A}_{x} \mathrm{MnO}_{3}$, the potential fluctuation experienced by the $e_{g}$ electrons due to the $\mathrm{La}^{3+}$ and $\mathrm{A}^{2+}$ ion cores, if unscreened, would amount to $U \sim 0.6$ $\mathrm{eV}$. This is equivalent to $W \approx 0.6[12 x(1-x)]^{1 / 2} \mathrm{eV}=$ $0.95 \mathrm{eV}$ at $\mathrm{x}=0.3$. Using Gutzwiller approximation [7] the band narrowing from e-e interactions at $V=20 t$ is estimated to be around 0.5 for $x=0.2$ and 0.6 for $x=0.3$, if the two orbitals are degenerate. Hence $W / t_{\text {eff }}$ can be as large as 13 , if we assume $\mathrm{t}=0.15 \mathrm{eV}$. Screening certainly will reduce this value, so our estimate is only meant to stress the importance of disorder in MIT in Mn-oxides. We feel that disorder in CMR materials is not strong enough to induce localization by itself, but its sizable presence can certainly modify small polaron formation picture [6, ]7 for systems with strong but less than criti- cal value of electron-phonon coupling. This mechanism also has the advantage of not restricting to any particular form of electron-phonon coupling, and thus may apply to a wide range of CMR materials described by the double exchange mechanism.

In summary, we have investigated the charge localization properties in the Mn-oxides in the classical spin limit. We find that a substantial amount of diagonal disorder is required to localize $20-30 \%$ of electronic states. This suggests that large electron-phonon coupling and polaronic effect are necessary to explain the MIT close to $T_{c}$.

We would like to thank S.A. Trugman and H. Röder for helpful discussions. Ames Laboratory is operated for the U.S. Department of Energy by Iowa State University under Contract No. W-7405-Eng-82. This work was supported by the directorate for Energy Research, Office of Basic Energy Sciences. Work at Los Alamos is performed under the auspices of the U.S. DOE.

[1] R. M. Kusters et al, Physica (Amsterdam) 155B, 362 (1989); K. Chahara et al., Appl. Phys. Lett. 71, 1990 (1993); R. von Helmholt et al, Phys. Rev. Lett. 71, 2331 (1993); S. Jin et al., Science 264, 413 (1994).

[2] G.H. Jonker and J.H. Van Santen, Physica 16, 337 (1950); ibid 19, 120 (1950); J. Volger, Physica 20, 4966 (1954); E.D. Wollan and W.C. Koehler, Phys. Rev. 100, 545 (1955).

[3] C. Zener, Phys. Rev. 82, 403 (1951); P . W. Anderson and H. Hasegawa, Phys. Rev. 100, 675 (1955); P.G. de Gennes, Phys. Rev. 82, 403 (1951).

[4] A. J. Millis, P. B. Littlewood, and B. I. Shraiman, Phys. Rev. Lett. 74, 5144 (1995).

[5] J.B. Goodenough, Phys. Rev. 100, 564 (1955); G. Matsumoto, J. Phys. Soc. Japan, 29, 615, (1970).

[6] A. J. Millis, B. I. Shraiman, and R. Mueller, Phys. Rev. Lett.77, 175 (1996); and A. J. Millis et al Phys. Rev. B (1996)

[7] H. Röder, Jun Zang, and A. R. Bishop, Phys. Rev. Lett. 76, 1356 (1996); Jun Zang, A. R. Bishop, and H. Röder, Phys. Rev. B. 53, R8840 (1996).

[8] N. Furukawa, J. Phys. Soc. Jpn. 63, 3214 (1994).

[9] C. M. Varma, Phys. Rev. B 54, 7328 (1996).

[10] T.A. Tyson et al, Phys. Rev. B 53, 13985 (1996); P. Dai et al, Phys. Rev. B 54, R3694 (1996); S. J. L. Billinge et al. Phys. Rev. Lett. 77, 715 (1996); S.G. Kaplan et al, Phys. Rev. Lett. 772081 (1996).

[11] G. M. Zhao et al, Nature 381676 (1996); M. Jaime et al, Appl. Phys. Lett. March, 1996.

[12] For a recent review, see B. Kramer and A. MacKinnon, Rep. Progr. Phys. 56, 1469 (1993).

[13] J. Mazzaferro, C.A. Balsero, and B. Alascio, J. Phy. Chem. Soli. 46, 1339 (1985); J.M.D. Coey et al, Phys. Rev. Lett. 75, 3910 (1995). 
[14] M.E. Fisher and J.S. Langer, Phys. Rev. Lett. 20, 665 (1968).

[15] C. M. Soukoulis et al, Phys. Rev. B26, 1838 (1982); C. M. Soukoulis and E. N. Economou, Phys. Rev. B24, 5698 (1981); Qiming Li, C. M. Soukoulis, and E. N. Economou, Phys. Rev. B37, 8289 (1988); M. Inui, S.A. Trugman, E. Abrahams, Phys. Rev. B 49, 3190 (1994).

[16] For a review, see B. Huckestein, Rev. Mod. Phys. 67, 357 (1995).

[17] E. N. Economou and P. D. Antoniou, Sol. State Comm. 21, 285 (1977).

[18] D. C. Licciardello and E. N. Economou, Phys. Rev. B11, 3697 (1975).

[19] R. Allub and B. Alacio, Sol. State Comm. 99, 613 (1996); and unpublished (cond-mat/9608086).

[20] J. M. Ziman, J. Phys. C 2, 1230 (1969).

[21] This behavior should be distinguished from the localization effect due to purely random phases in otherwise periodic systems, for example, in the random flux problem that has been extensively investigated recently 16 .

[22] E. N. Economou. C. M. Soukoulis, and A. D. Zdetsis, Phys. Rev. B30, 1686 (1984); and A. D. Zdetsis, C. M. Soukoulis, E. N. Economou. and G. S. Grest. Phys. Rev. B32, 8711 (1985).

[23] The true band edge, according to the Lifshitz argument, is located at $\pm(6 t+W / 2)$.

[24] A. Sumi and Y. Toyozawa, J. Phys. Soc. Jpn. 35, 137 (1973).

[25] M. H. Cohen, E. N. Economou, and C. M. Soukoulis, Phys. Rev. Lett. 51, 1202 (1983).

[26] M. Henneke, B. Kramer, and T. Phtsuki. Europhys. Lett. 27, 389 (1994).

[27] E. Hofstetter and M. Schreiber, Phys. Rev. Lett. 73, 3137 (1994).

FIG. 1. The ratio of the finite size localization length to the width, $\lambda_{M} / M$, as a function of $\mathrm{M}$, for the double exchange model in the absence of any diagonal disorders. The mobility edge is located around $E_{c}=-3.56 t$. All states with $E<E_{c}$ are localized.

FIG. 2. Th integrated density of states of the double exchange model in the absence of any diagonal disorder, $\mathrm{W}=$ 0 . The density of states (in units of $t^{-1}$ ) is shown in the inset. The results are obtained from exact diagonalization of $10 \times 10 \times 10$ clusters averaged over 100 random spin configurations.

FIG. 3. The mobility edge trajectory of the double exchange model with diagonal disorder $\mathrm{W}$ for electron-phonon coupling canstant $\lambda=0,0.03$, and 1 . The dashed lines show equal integrated density of states lines at 10\%,20\%,30\%, and $40 \%$ fractions. The density of states and the mobility edge trajectory are symmetric around $\mathrm{E}=0$ axis.
FIG. 4. The universal scaling function of the double exchange model for different $\mathrm{W}, \mathrm{E}$, and $\mathrm{M}$. At the critical point, $\lambda_{M} / M \approx 0.6$. The solid line is the universal scaling curve for the standard Anderson model with diagonal disorder alone. 


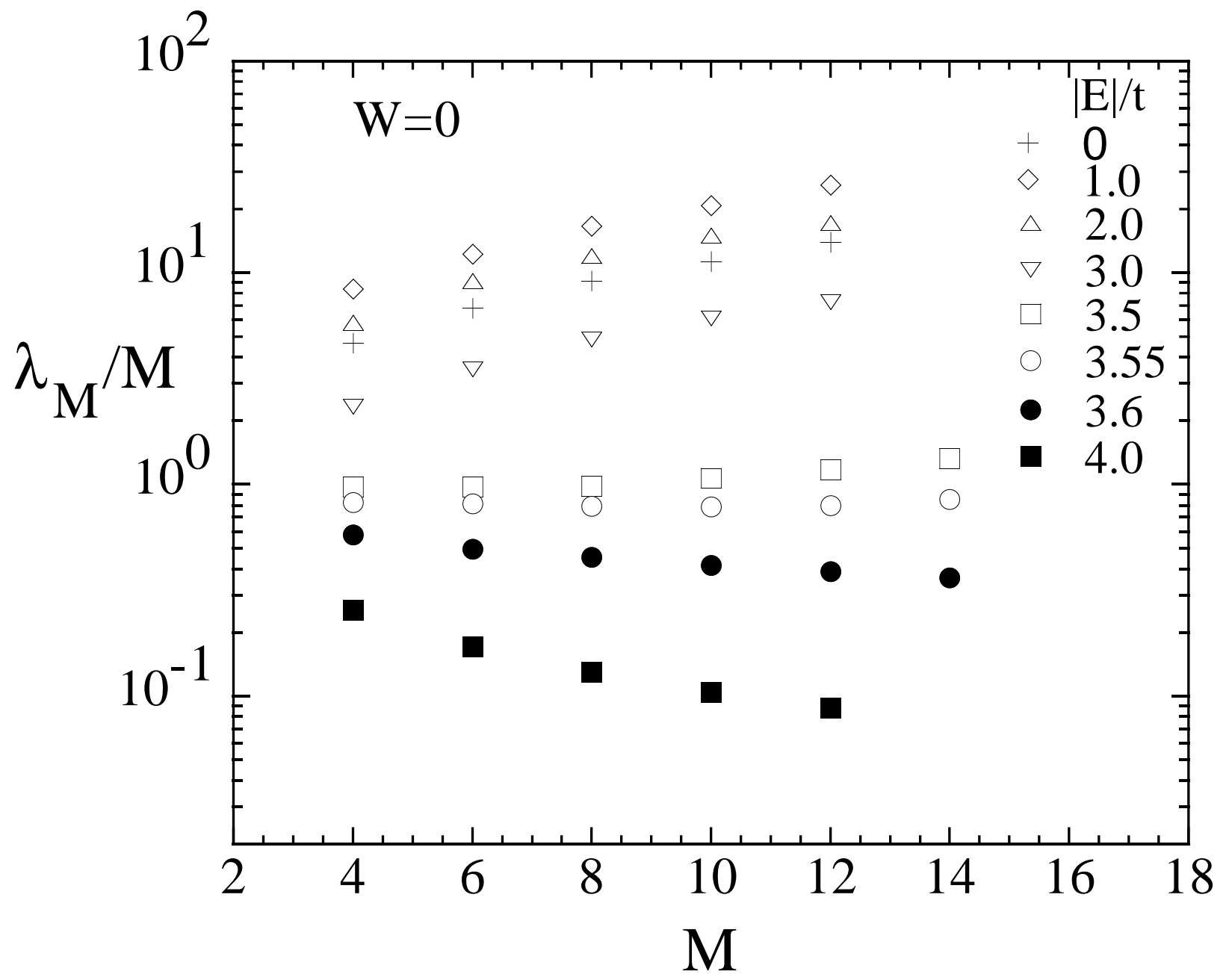




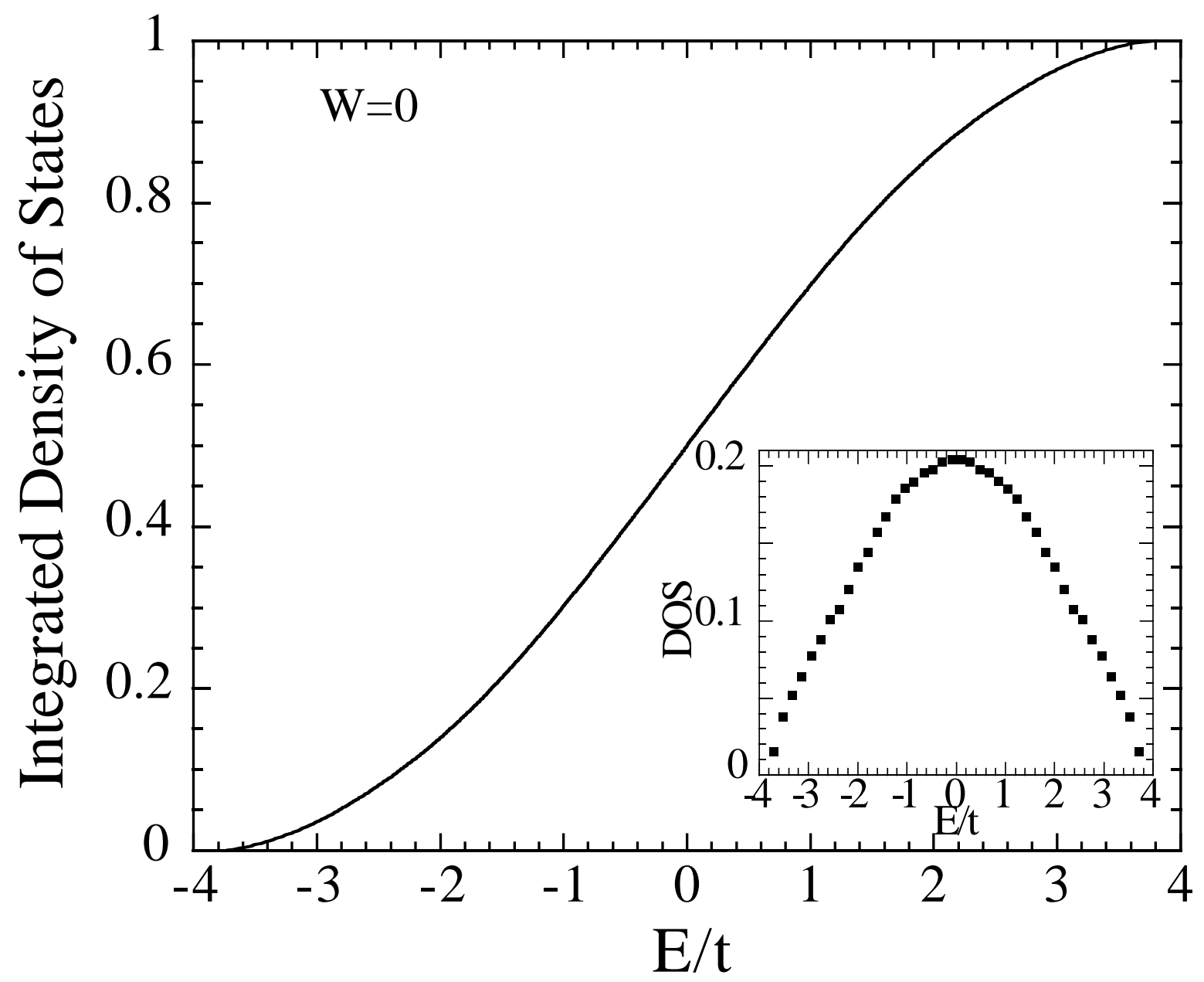




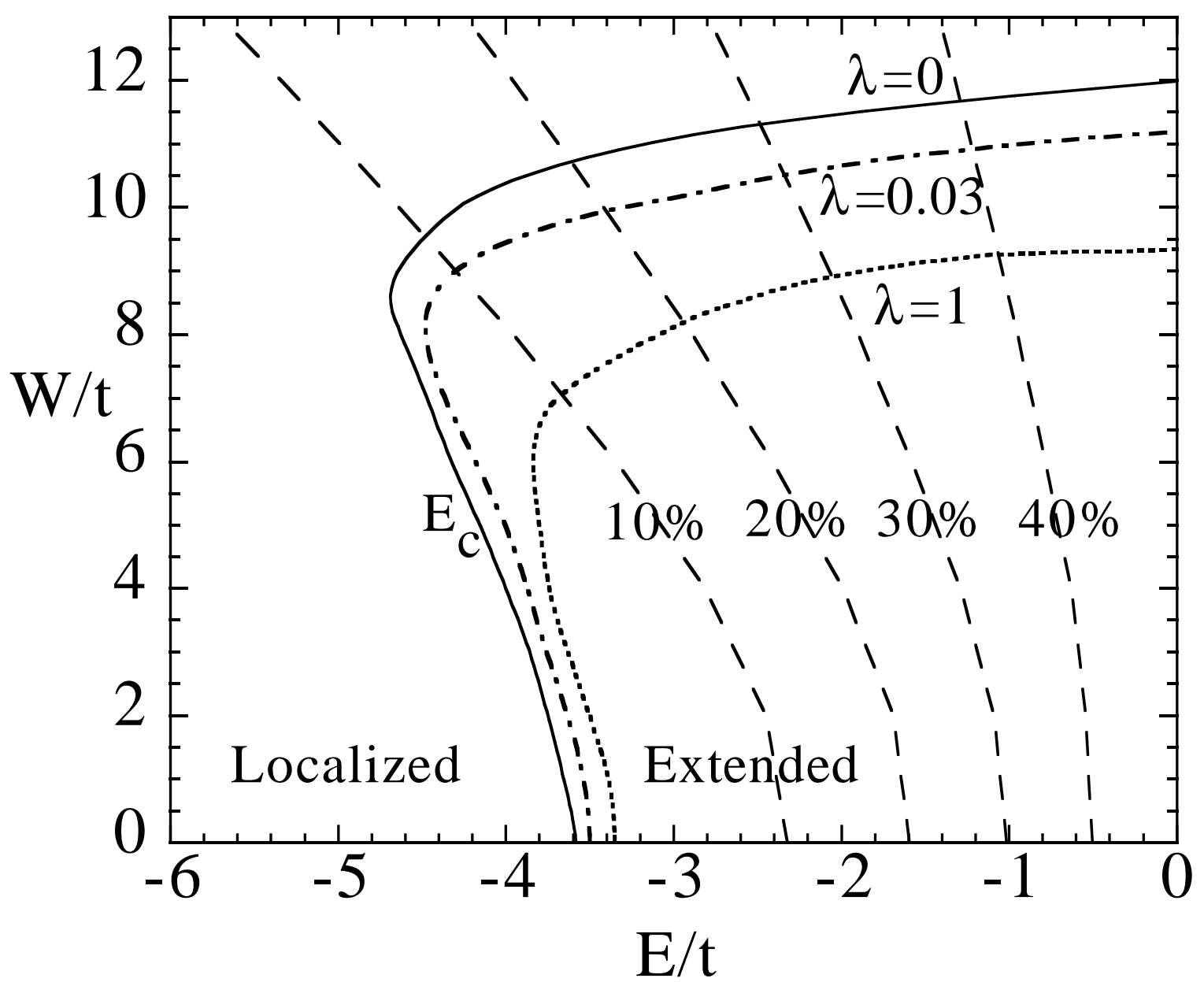




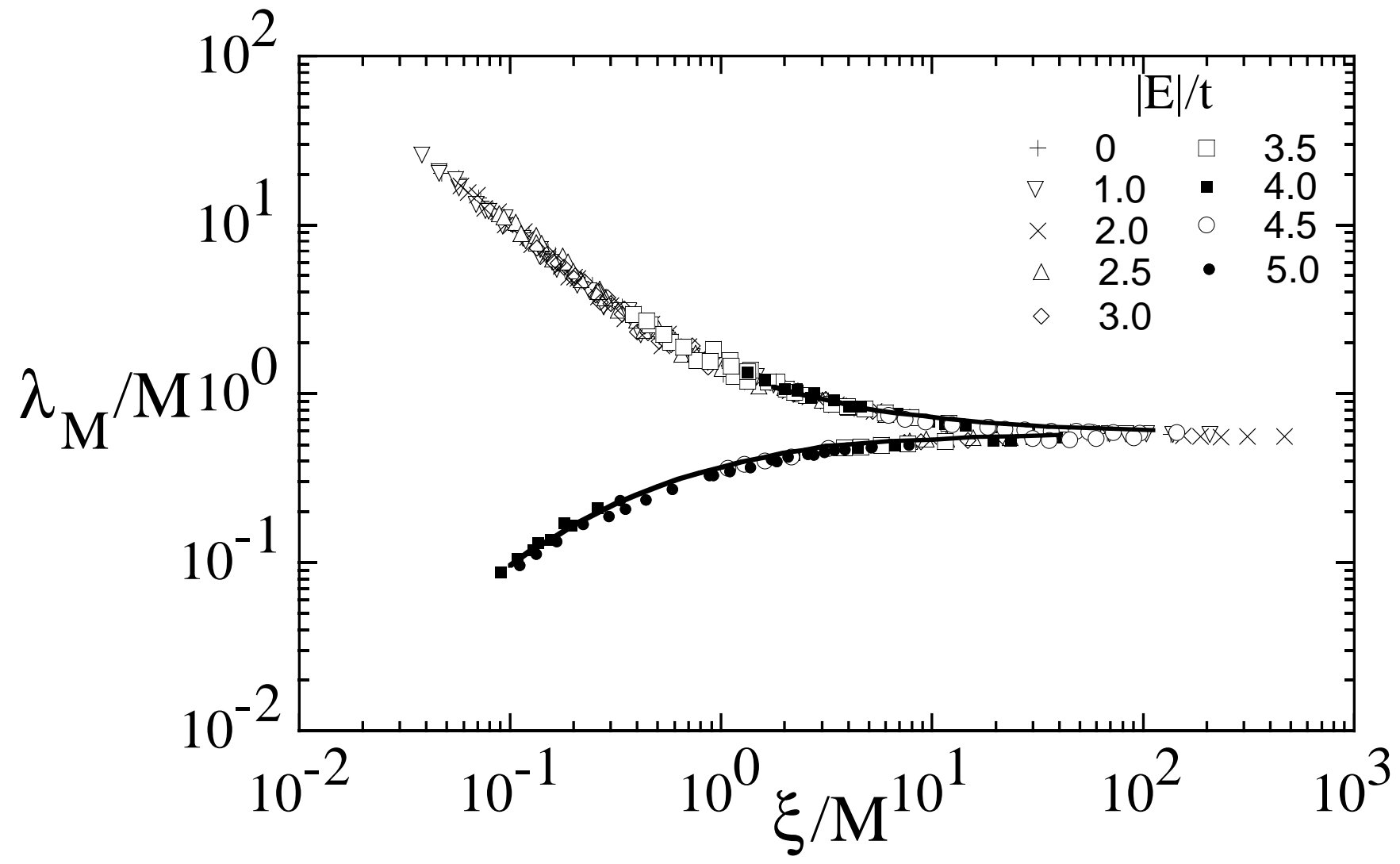

